\title{
ANÁLISE DE CORRELAÇÃO ENTRE FORÇA MUSCULAR, IGF-1 E AUTONOMIA FUNCIONAL EM IDOSAS COM EXCESSO DE PESO SUBMETIDAS A EXERCÍCIOS RESISTIDOS AQUÁTICOS
}

Juliana Brandão Pinto de Castro ${ }^{1}$, Rosana Dias de Oliveira Brum², Carlos Soares Pernambuco ${ }^{2}$, Rodrigo Gomes de Souza Vale ${ }^{1,2}$

${ }^{1}$ Programa de Pós-Graduação em Ciências do Exercício e do Esporte, Universidade do Estado do Rio de Janeiro, Rio de Janeiro, RJ, Brasil ${ }^{2}$ Laboratório de Fisiologia do Exercício, Universidade Estácio de Sá, Cabo Frio, RJ, Brasil

\section{OPEN ACCES \\ Correspondencia: \\ Rodrigo Gomes de Souza Vale Universidade do Estado do Rio de Janeiro, Rua São Francisco Xavier, 524, Pavilhão João Lira Filho, 9o andar, Bloco F, sala 9122, CEP: 20550-900, Maracanã, Rio de Janeiro, RJ, Brasil. \\ E-mail: rodrigovale@globo.com \\ Funciones de los autores: Todos los autores trabajaro equitativamente en la consecución de \\ trabajo. \\ Recibido: $23 / 04 / 2018$ Aceptado: 10/11/2018 Publicado: 31/01/2019 \\ Citación: \\ Pinto de Castro, J.B., Oliveira-Brum, $\mathrm{R}$, Soares-Pernambuco, C., \& Gomes de Souza-Vale, R. (2019). Análise de correlação entre força muscular, IGF-1 autonomia funcional em idosas com excesso de peso submetidas a exercícios resistidos aquáticos. RIAA. Revista de Investigación en Actividades Acuáticas,3(5), 18-23. https://doi.org/10.21134/riaa.v3i5.1575}

@creative (c) (1)(2)

Creative Commons License Esta obra está bajo una licencia de Creative Commons ReconocimientoNoComercial-Compartir-Igua 4.0 Internacion
Resumo

Contexto: Dentre as alterações orgânicas que tendem a ocorrer com o envelhecimento, tem-se a redução da força muscular, dos níveis séricos de IGF-1 e da autonomia funcional (AF). Contudo, esses efeitos deletérios podem ser amenizados por meio da prática de exercícios físicos, como a hidroginástica.

Objetivos: Analisar a correlação entre força muscular, IGF-1, IGFBP3 e AF em idosas com excesso de peso submetidas a exercícios resistidos aquáticos.

Método: A amostra foi composta por 14 idosas (idade: $68,93 \pm 4,46$ anos; IMC: $28,55 \pm 2,08 \mathrm{~kg} / \mathrm{m}^{2}$ ) submetidas a exercícios resistidos aquáticos durante 12 semanas. A intervenção ocorreu 3 vezes por semana, com 50 minutos por sessão de treinamento. A força muscular foi mensurada através de dinamometria, incluindo força de membros inferiores e de preensão palmar direita (FMD) e esquerda (FME). A coleta de sangue para análise do IGF-1 e seu principal carreador, o IGFBP3, foi realizada através do método quimioluminescência. A AF foi avaliada por meio do protocolo de autonomia GDLAM.

Resultados: $O$ teste de correlação de Pearson apresentou correlação ( $r$ ) positiva e significativa entre FMD e FME, FMD e IGF1, FMD e IGFBP3, FME e IGF1, FME e IGFBP3, e IGF1 e IGFBP3.

Conclusoes: A prática de exercícios resistidos aquáticos pode melhorar os níveis de força muscular de preensão manual, de IGF1 e de IGFBP3 em idosas com excesso de peso.

Palavras-chave: atividades aquáticas, idoso, atividades da vida diária, treinamento de resistência.

Titulo: Análisis de correlación entre fuerza muscular, IGF-1 y autonomía funcional en ancianas con exceso de peso sometidas a ejercicios resistidos acuáticos

Antecedentes: Entre las alteraciones orgánicas que tienden a ocurrir con el envejecimiento, se tiene la reducción de la fuerza muscular, de los niveles séricos de IGF-1 y de la autonomía funcional (AF). Sin embargo, estos efectos deletéreos pueden ser amenizados por medio de la práctica de ejercicios físicos, como la hidrogimnasia.

Objetivos: Analizar la correlación entre fuerza muscular, IGF-1, IGFBP3 y AF en ancianas con exceso de peso sometidas a ejercicios resistidos acuáticos

Método: La muestra fue compuesta por 14 ancianas (edad: $68,93 \pm 4,46$ años; IMC: $28,55 \pm 2,08 \mathrm{~kg} / \mathrm{m}^{2}$ ) sometidas a ejercicios resistidos acuáticos durante 12 semanas. La intervención ocurrió 3 veces por semana, con 50 minutos por sesión de entrenamiento. La fuerza muscular se midió a través de dinamometría, incluyendo fuerza de miembros inferiores y de agarre derecha (FMD) e izquierda (FME). La recolección de sangre para análisis del IGF-1 y su principal cargador, el IGFBP3, fue realizada a través del método quimioluminescencia. La AF fue evaluada por medio del protocolo de autonomía GDLAM.

Resultados: A través del procedimiento estadístico de correlación de Pearson, se verificó una correlación ( $r$ ) positiva y significativa entre FMD y FME, FMD e IGF1, FMD e IGFBP3, FME e IGF1, FME e IGFBP3, e IGF1 e IGFBP3.

Conclusiones: La práctica de ejercicios resistidos acuáticos puede mejorar los niveles de fuerza muscular de agarre manual, de IGF1 y de IGFBP3 en mujeres mayores con sobrepeso.

Palabras clave: actividades acuáticas, ancianos, actividades de la vida diaria, entrenamiento de resistencia.

Title: Analysis of correlation between muscle strength, IGF-1 and functional autonomy in overweight elderly women submitted to aquatic resistance exercises

Background: Among the organic changes that tend to occur with aging, there is a reduction of muscle strength, serum IGF-1 levels and functional autonomy (FA). However, these deleterious effects can be mitigated through the practice of physical exercises, such as hydrogymnastics.

Objectives: To analyze the correlation between muscle strength, IGF-1, IGFBP3 and FA in overweight elderly women submitted to aquatic resistance exercises.

Method: The sample consisted of 14 elderly women (age: $68.93 \pm 4.46$ years; BMI: $28.55 \pm 2.08 \mathrm{~kg} / \mathrm{m}^{2}$ ) submitted to aquatic resistance exercises for 12 weeks. The intervention occurred 3 times a week, with 50 minutes per training session. Muscle strength was measured by dynamometry, including lower limb strength and right (RPS) and left (LPS) palmar grip strength. Blood collection for the analysis of IGF-1 and its main carrier, the IGFBP3, was performed using the chemiluminescence method. FA was assessed through the GDLAM autonomy protocol. Results: The Pearson correlation test presented a positive and significant correlation coefficient ( $r$ ) between RPS and LPS, RPS and IGF1, RPS and IGFBP3, LPS and IGF1, LPS and IGFBP3, and IGF1 and IGFBP3.

Conclusions: The practice of aquatic resistance exercises can improve the muscle strength of manual grip, IGF1 and IGFBP3 levels in overweight elderly women.

Key words: aquatic activities, elderly, activities of daily living, resistance training. 


\section{Introdução}

O processo de envelhecimento costuma vir acompanhado de alterações estruturais e funcionais que podem repercutir negativamente na capacidade funcional de idosos (ACSM et al., 2009). Dentre as alterações, destacam-se os déficits de equilíbrio, mobilidade, flexibilidade e força muscular (Locks et al., 2012), anormalidades endócrinas - como a redução dos níveis séricos do fator de crescimento insulina símile-1 (Insulin-Like Growth Factor I - IGF-1) (Eliakim, Oh, \& Cooper, 2000; Fornelli, Isaia, \& D'Amelio, 2016) - e modificações na composição corporal, incluindo diminuição tanto da massa muscular quanto da massa óssea (Frontera et al., 2000), além de aumento na prevalência de obesidade (Jura \& Kozak, 2016).

O IGF-1 está ligado principalmente ao transportador denominado proteína de ligação semelhante à insulina-3 (Insulin-Like Binding Protein-3 - IGFBP-3) (Grimberg \& Cohen, 2000). A redução da secreção de IGF-1 e do IGFBP-3 decorrente do envelhecimento pode estar associada à perda de massa magra e força muscular (Cappola, BandeenRoche, Wand, Volpato, \& Fried, 2001), à diminuição da síntese de proteína e ao aumento da massa adiposa (Friedrich et al., 2009; Oliveira, Côrtes, Vale, \& Dantas, 2003). O principal regulador do IGF-1 é o hormônio de crescimento (Growth Hormone - GH). Ambos são considerados importantes agentes anabólicos proteicos para a homeostase corporal (Frystyk, 2010).

Além desses declínios hormonais, a redução de força muscular apresenta-se como um grande problema enfrentado pelo idoso, comprometendo a autonomia funcional (AF) (Mariano, Navarro, Sauaia, Oliveira Junior, \& Marques, 2013; Vale, Novaes, \& Dantas, 2005). Tal déficit é originado, dentre outros fatores, pela diminuição da massa e da função muscular associada ao envelhecimento - a sarcopenia, que, por consequência, pode ocasionar o excesso de peso (Kamel, 2003; Rosenberg, 1997).

A realização de exercícios físicos contribui para o aprimoramento da AF de idosos e melhoria no desempenho em atividades da vida diária (AVD), tais como sentar-se e levantar-se com mais facilidade, vestir-se sozinho e caminhar mais rapidamente (Vale et al., 2018; Mazini Filho et al., 2018; Daniel, Vale, Giani, Bacellar, \& Dantas, 2012).

Maiores níveis de IGF-1 trazem benefícios a idosas, sendo relevante o conhecimento de meios que aumentem sua síntese e liberação (Castro \& Vale, 2017; Oliveira et al., 2003). O treinamento de força auxilia no incremento dos níveis hormonais de IGF-1 (Vale et al., 2017a; Vale \& Rodrigues, 2014; Bastos et al., 2013; Vale et al., 2009). Os exercícios no meio aquático podem ser uma alternativa aos exercícios terrestres, apresentando menos estresse nas articulações e menor risco de quedas quando comparado ao ambiente terrestre (Hale, Waters, \& Herbison, 2012). Nesse sentido, um exercício físico que costuma ser praticado pela população idosa é a hidroginástica (Lima et al., 2017).

Portanto, o objetivo do presente estudo foi analisar a correlação entre força muscular, IGF-1 e AF em idosas com excesso de peso submetidas a exercícios resistidos aquáticos.

\section{Método}

\section{Participantes}

Este estudo é do tipo quase-experimental de corte transversal. O grupo amostral foi composto por 14 idosas voluntárias com excesso de peso. Os critérios de inclusão foram: a) mulheres com idade igual ou superior a 60 anos; b) estar apta fisicamente para participar da intervenção; c) ser independente no desempenho das AVD; d) estar participando da atividade de hidroginástica regularmente há pelo menos três meses; e) apresentar índice da massa corporal (IMC) acima de $25 \mathrm{~kg} / \mathrm{m}^{2}$; f) ser considerada apta após avaliação médica, autorizando a prática de atividade física.

Os critérios de exclusão foram: a) apresentar qualquer tipo de condição aguda ou crônica que pudesse comprometer o desempenho ou que se tornasse um fator de impedimento para a participação de um programa de hidroginástica, tais como cardiopatias, diabetes, hipertensão arterial e asma não controladas; b) quaisquer condições musculoesqueléticas que pudessem servir de fator interveniente à prática da hidroginástica (osteoartrite, fratura recente, tendinite e uso de prótese).

As idosas que concordaram em participar voluntariamente do estudo assinaram o termo de consentimento livre e esclarecido (TCLE), respeitando as normas éticas da Resolução no 466/2012 do Conselho Nacional de Saúde para pesquisas envolvendo seres humanos.

\section{Procedimentos de coleta de dados}

\section{Avaliação antropométrica}

Com objetivo de caracterização da amostra, foram realizadas as medidas de massa corporal, da estatura e cálculo do IMC. Para avaliação da massa corporal e da estatura, foi utilizada uma balança mecânica (Filizola ${ }^{\circ}$, Brasil), precisão de 100 gramas e capacidade para $150 \mathrm{~kg}$, com estadiômetro, precisão de $0,5 \mathrm{~cm}$. Todas medidas foram realizadas por um único avaliador, conforme protocolo da International Standards for Anthropometric Assessment (Marfell-Jones et al., 2006). O cálculo do IMC foi realizado com base na divisão da massa corporal, em quilogramas, pelo quadrado da estatura, em metros $\left(\mathrm{kg} / \mathrm{m}^{2}\right)$.

\section{Força muscular}

Foram feitas medidas de força muscular de membros inferiores e de preensão palmar direita e esquerda, através dos dinamômetros dorsal e de preensão manual (Takei ${ }^{\circledR}$ - Tóquio, Japão), respectivamente, adotando a unidade de medida em quilogramas $(\mathrm{kg})$. No teste com o dinamômetro dorsal, o avaliado posicionou-se em pé sobre o equipamento e com os joelhos flexionados em aproximadamente 120으 e tronco ereto. A barra de tração foi posicionada à altura da prega inguinal. $O$ teste é realizado com a extensão dos joelhos sem flexionar o tronco (Johnson \& Nelson, 1979). Durante a realização do teste de preensão manual, a idosa permaneceu em pé com o braço estendido ao lado do corpo e punho em rotação neutra. A empunhadura foi ajustada para cada indivíduo (Desrosiers, Bravo, Hébert, \& Dutil, 1995). No início dos testes, a agulha foi colocada na posição neutra (zero). Em ambos os testes, ao comando de voz do avaliador, a idosa deveria realizar o máximo de força. Após essa tentativa, o examinador registrou a leitura da força aplicada pelo indivíduo. Foram coletadas três medidas, utilizando-se o maior valor obtido. $O$ intervalo de descanso entre uma tentativa e outra foi de um minuto (Hillman et al., 2005).

\section{IGF-1 sérico}

Para a análise dos níveis séricos de IGF-1 e IGFBP3, foi aplicado o método de ensaio imunorradiométrico pós-extração, com kit comercial de quimioluminescência. As amostras de sangue foram obtidas pela manhã, onde não foi necessário jejum completo. Apenas evitou-se a ingestão de leite e derivados. Para dosagem do IGF-1 e IGFBP3, as idosas permaneceram em repouso. As amostras de sangue, no mínimo $5 \mathrm{ml}$, foram colhidas em tubo seco e o soro separado e enviado para processamento. A coleta foi feita em punção venosa. Em seguida, o sangue foi centrifugado a $3.500 \mathrm{rpm}$ por 10 minutos para obter no mínimo $1 \mathrm{ml}$ de soro. $O$ soro cadastrado foi colocado no equipamento IMMULITE - DPC, sistema a vácuo, no rack de amostras. Foi feita a análise por quimioluminescência num período de 3 minutos por amostra. O resultado foi impresso ou passado direto ao computador central, por interfaceamento. Os valores de referência utilizados para o 
IGF1 $(69-200 \mathrm{ng} / \mathrm{ml})$ para análise foram obtidos segundo recomendações de Huayllas et al. (2001)

\section{Autonomia funcional}

A AF foi analisada por intermédio do protocolo GDLAM de autonomia funcional (Dantas \& Vale, 2004; Dantas, Figueira, Emygdio, \& Vale, 2014), constituído de caminhar 10m (C10m) (Sipilä, Multanen, Kallinen, Era, \& Suominen, 1996), levantar-se da posição sentada (LPS) (Guralnik et al., 1994), levantar-se da posição decúbito ventral (LPDV) (Alexander et al., 1997), levantar-se da cadeira e locomover-se pela casa (LCLC) (Andreotti \& Okuma, 1999) e o teste de vestir e tirar uma camiseta (VTC) (Vale, Pernambuco, Novaes, \& Dantas, 2006). As participantes executaram duas tentativas em cada teste, com intervalo de cinco minutos entre cada tentativa, em um ambiente adequado. Registrou-se o menor tempo, em segundas, através de um cronômetro (Casio, Brasil). Após a mensuração do tempo de cada teste, foi calculado o índice GDLAM de autonomia (IG), em escores (Vale \& Dantas, 2008), através da seguinte fórmula:

$$
I G=\frac{[(C 10 m+L P S+L P D V+V T C) \times 2]+L C L C}{4}
$$

Onde C10m, LPS, LPDV, VTC e LCLC correspondem ao tempo aferido em segundos e IG é o índice GDLAM em escores. Quanto menor o escore, melhor o resultado.

\section{Intervenção}

O programa de treinamento foi constituído de exercícios resistidos de hidroginástica numa piscina aquecida ( 27 a $29^{\circ} \mathrm{C}$ ) com profundidade de 1.00 a $1.40 \mathrm{~m}$, em que foi exigido um posicionamento dos sujeitos de forma que a lâmina da água se mantivesse na linha axilar. A intervenção foi dividida em: 1 ) fase básica (quatro semanas; 3 x 15-20 repetições; sem o uso de acessórios aquáticos); e 2 ) fase específica (oito semanas; 3 × 8-10 repetições; com utilização de acessórios aquáticos). O programa de hidroginástica durou 12 semanas e consistiu em 3 sessões semanais em dias alternados. Cada sessão foi composta por aquecimento (10 minutos), parte principal (35 minutos) e volta à calma (5 minutos), totalizando 50 minutos de duração. O aquecimento envolveu exercícios de caminhada, passadas laterais no step, alternando diferentes movimentos de membros superiores e inferiores. A parte principal consistiu em três séries de 8 a 10 repetições com um período de descanso de 30 segundos a um minuto entre séries, utilizando acessórios específicos para exercícios aquáticos (halteres, caneleiras e luvas) a fim de aumentar o grau de esforço das sessões de treinamento. Os exercícios foram realizados com alternância dos segmentos corporais, incluindo: flexão e extensão horizontal do ombro na linha d'água, flexão e extensão do joelho, adução e abdução do ombro na direção oblíqua, flexão plantar e abdominais. A volta à calma consistiu em exercícios de alongamento em níveis submáximos. A intensidade de treinamento da parte principal foi controlada pela manutenção da escala OMNI-RES de esforço percebido (Robertson et al., 2003) entre os níveis 3 e 5 (intensidade leve a média) nas primeiras quatro semanas e entre 5 e 7 (intensidade forte) até o final do período de intervenção. Na volta à calma, realizou-se exercícios de alongamento em níveis submáximos controlados por meio da escala de esforço percebido na flexibilidade (Scale of perceived exertion in the flexibility - PERFLEX) (Dantas et al., 2008), que indicou nível de esforço entre os escores 31 e 60 .

\section{Analises estatística}

Os dados foram apresentados de forma descritiva como média, desviopadrão, e valores mínimos e máximos. A normalidade dos dados foi verificada pelo teste de Shapiro-Wilk. O teste de correlação de Pearson foi empregado para analisar as possíveis associações entre as variáveis de estudo. $O$ nível de $p<0.05$ foi adotado para a significância estatística.
Os dados foram processados no software Statistical Package for Social Sciences (SPSS, versão 20.0 - Chicago, USA).

\section{Resultados}

A tabela 1 apresenta as características gerais da amostra. Observa-se que o grupo amostral apresentou uma distribuição próxima da curva normal, visto os valores de significância $(p>0.05)$ encontrados no teste de Shapiro-Wilk em todas as variáveis.

Tabela 1. Características gerais da amostra $(n=14)$.

\begin{tabular}{lccccc}
\hline & Média & DP & Mínimo & Máximo & $\begin{array}{c}\text { Valor-p } \\
(\mathrm{SW})\end{array}$ \\
\hline $\begin{array}{l}\text { Idade (anos) } \\
\text { Massa }\end{array}$ & 68.93 & 4.46 & 62.00 & 79.00 & 0.834 \\
corporal $(\mathrm{kg})$ & 71.06 & 5.03 & 59.70 & 78.30 & 0.228 \\
Estatura $(\mathrm{m})$ & 1.58 & 0.05 & 1.48 & 1.64 & 0.081 \\
$\mathrm{IMC}\left(\mathrm{kg} / \mathrm{m}^{2}\right)$ & 28.55 & 2.08 & 25.28 & 32.37 & 0.776 \\
\hline Nota: DP = desvio padrão; IMC = índice de massa corporal; SW = Shapiro-Wilk.
\end{tabular}

Na tabela 2, encontram-se os resultados descritivos dos testes de força muscular de preensão manual direita e esquerda, e dos níveis séricos de IGF-1 e IGFBP-3. Verificando-se os resultados destas variáveis, observa-se que os níveis de força muscular são semelhantes entre as mão direita e esquerda. Além disso, todas as variáveis apresentaram normalidade em seus dados.

Tabela 2. Resultados dos testes de força muscular e dos níveis séricos de IGF-1 e IGFBP-3.

\begin{tabular}{lccccc}
\hline Variáveis & Média & DP & Mínimo & Máximo & $\begin{array}{c}\text { Valor-p } \\
(\mathrm{SW})\end{array}$ \\
\hline FMD (kg) & 29.64 & 4.99 & 23.00 & 37.00 & 0.132 \\
FME (kg) & 29.29 & 4.56 & 22.00 & 36.00 & 0.396 \\
FMMII (kg) & 97.86 & 14.76 & 76.00 & 128.00 & 0.874 \\
IGF1 (ng/ml) & 116.78 & 12.38 & 98.20 & 134.00 & 0.353 \\
$\begin{array}{l}\text { IGFBP3 } \\
\text { (ng/ml) }\end{array}$ & 3.54 & 0.75 & 2.10 & 4.60 & 0.442 \\
\hline
\end{tabular}

Nota: $\mathrm{DP}$ = desvio padrão; $\mathrm{SW}=$ Shapiro-Wilk; FMD = força de preensão manual direita; FME = força de preensão manual esquerda; $F M M I I$ = força de membros inferiores; IGF1 = fator de crescimento insulina símile-1; IGFBP3 = proteína de ligação semelhante à insulina-3.

Os resultados dos testes de AF estão na tabela 3. Observando-se estes resultados, verifica-se que os níveis de desempenho nas AVD da amostra são semelhantes.

Tabela 3. Resultados descritivos dos testes de autonomia funcional.

\begin{tabular}{lccccc}
\hline & Média & DP & Mínimo & Máximo & Valor-p (SW) \\
\hline C10M & 6.03 & 1.02 & 4.87 & 8.24 & 0.119 \\
LPS & 10.01 & 0.84 & 8.47 & 12.00 & 0.498 \\
LPDV & 2.85 & 0.55 & 2.13 & 3.97 & 0.083 \\
VTC & 9.81 & 1.90 & 6.88 & 13.79 & 0.627 \\
LCLC & 42.31 & 4.08 & 37.35 & 50.44 & 0.229 \\
IG & 24.93 & 2.65 & 21.93 & 30.68 & 0.102 \\
\hline
\end{tabular}

Nota: $\mathrm{DP}=$ desvio padrão; $\mathrm{SW}=$ Shapiro-Wilk; $\mathrm{C10 \textrm {m }}=$ caminhar 10 metros; $\mathrm{LPS}=$ levantar da posição sentada; LPDV = levantar da posição decúbito ventral; VTC = vestir e tirar a camisa; LCLC = levantar da cadeira e locomover-se pela casa. Tempo em segundos. IG = índice GDLAM, em escores. 
A tabela 4 exibe os resultados da correlação de Pearson entre as variáveis dos testes de força, de AF e dos níveis hormonais coletados. Observou-se que houve correlação positiva e significativa entre FMD e FME, entre FMD e FME e IGF1 e IGFBP3, e entre IGF1 e IGFBP3. Isso representa que quanto maior a força muscular de preensão manual, maior foi a expressão do IGF1 e seu principal carreador, o IGFBP3. Não houve correlação entre as demais variáveis de estudo.

Tabela 4. Análise de correlação entre as variáveis de estudo analisadas.

\begin{tabular}{|c|c|c|c|c|c|c|}
\hline & & FMD & FME & FMMII & IGF1 & IGFBP3 \\
\hline \multirow[t]{2}{*}{ FME } & $r$ & $0.924^{*}$ & & & & \\
\hline & valor-p & $<0.000$ & & & & \\
\hline \multirow[t]{2}{*}{ FMMII } & r & 0.392 & 0.469 & & & \\
\hline & valor-p & 0.166 & 0.091 & & & \\
\hline \multirow[t]{2}{*}{ IGF1 } & r & $0.983^{*}$ & $0.887^{*}$ & 0.302 & & \\
\hline & valor-p & $<0.000$ & $<0.000$ & 0.293 & & \\
\hline \multirow[t]{2}{*}{ IGFBP3 } & r & $0.638^{*}$ & $0.582 *$ & 0.224 & $0.567^{*}$ & \\
\hline & valor-p & 0.014 & 0.029 & 0.442 & 0.035 & \\
\hline \multirow[t]{2}{*}{ C10M } & r & -0.100 & -0.078 & -0.474 & -0.073 & -0.065 \\
\hline & valor-p & 0.733 & 0.790 & 0.087 & 0.804 & 0.825 \\
\hline \multirow[t]{2}{*}{ LPS } & r & -0.092 & -0.109 & -0.228 & -0.101 & 0.272 \\
\hline & valor-p & 0.754 & 0.712 & 0.433 & 0.731 & 0.346 \\
\hline \multirow[t]{2}{*}{ LPDV } & r & -0.138 & -0.108 & -0.464 & -0.082 & -0.150 \\
\hline & valor-p & 0.638 & 0.714 & 0.095 & 0.781 & 0.609 \\
\hline \multirow[t]{2}{*}{ VTC } & r & -0.158 & -0.134 & -0.309 & -0.126 & -0.103 \\
\hline & valor-p & 0.589 & 0.647 & 0.283 & 0.668 & 0.726 \\
\hline \multirow[t]{2}{*}{ LCLC } & r & -0.208 & -0.219 & -0.274 & -0.203 & 0.009 \\
\hline & valor-p & 0.476 & 0.453 & 0.343 & 0.486 & 0.977 \\
\hline \multirow[t]{2}{*}{ IG } & r & -0.185 & -0.176 & -0.391 & -0.162 & -0.019 \\
\hline & valor-p & 0.527 & 0.548 & 0.167 & 0.580 & 0.949 \\
\hline
\end{tabular}
FMMII = força de membros inferiores; IGF1 = fator de crescimento insulina símile-1; IGFBP3 = proteína de ligação semelhante à insulina-3; C10m = caminhar 10 metros; LPS = levantar da posição sentada; LPDV = levantar da posição decúbito ventral; VTC = vestir e tirar a camisa; LCLC = levantar da cadeira e locomover-se pela casa; IG = índice GDLAM. ${ }^{*} p<0.05$

\section{Discussão}

Os resultados da presente investigação apresentaram correlações positivas e significativas entre FMD e FME, FMD e FME e IGF1 e IGFBP3, assim como entre o IGF1 e o IGFBP3. Isso demonstra que quanto maior a força de preensão manual, maiores são os níveis de IGF1 e de IGFBP3. Tais achados sugerem que o programa de exercício físico realizado proporcionou melhorias nas variáveis hormonais pesquisadas e na força muscular de preensão manual.

A força de preensão manual é importante para a realização das AVD, como segurar objetos, utilizar um corrimão ou apoios de transporte público, desempenhar tarefas domésticas, atividades de autocuidado, ou seja, realizar diferentes atividades cotidianas com autonomia. As idosas da presente pesquisa apresentaram melhoras na FMD e FME após a intervenção. Tal resultado mostra-se como relevante, uma vez que a força de preensão manual tem uma relação curvilínea com a idade (Wagner, Ascenço, \& Wibelinger, 2014).

O presente estudo utilizou o IMC para avaliar a composição corporal das idosas. Esse parâmetro é frequentemente utilizado em estudos epidemiológicos, em saúde pública e na área clínica, para classificação de baixo peso, sobrepeso e obesidade (WHO, 2000). Este índice é reconhecido como preditor de morbidades e mortalidade de diversas doenças crônicas não transmissíveis (DCNT) (Mota, Rinaldi, Pereira, Orsatti, \& Burini, 2011). As idosas investigadas apresentaram préobesidade $\left(25,00-29,99 \mathrm{~kg} / \mathrm{m}^{2}\right)$ e obesidade grau I $(30,00-34,99$ $\mathrm{kg} / \mathrm{m}^{2}$ ), representando um risco aumentado e moderado para as diversas comorbidades associadas ao excesso de peso (WHO, 2000).

Estudos de revisão sistemática e metanálise (Borde, Hortobágyi, \& Granacher, 2015; Steib, Schoene, \& Pfeifer, 2010) analisaram a relação dose-resposta do treinamento resistido em adultos mais velhos. Esses estudos apontam que a força muscular melhora substancialmente com o treinamento resistido realizado com intensidades de treinamento mais elevadas (Steib, Schoene, \& Pfeifer, 2010), sugerindo um período de treinamento de 50-53 semanas, com três sessões de treinamento por semana, com duas a três séries por exercício, 7 a 9 repetições por série e uma intensidade de treinamento de 51 a $69 \%$ do 1RM (Borde, Hortobágyi, \& Granacher, 2015). Apesar do tempo de intervenção e da frequência de treinamento ter sido inferior ao sugerido nesses estudos e de ter sido realizado em meio aquático ao invés de meio terrestre, foram encontradas melhoras nos níveis de força muscular de preensão manual no presente estudo. Desse modo, os resultados do presente estudo se mostram positivos, visto que maiores níveis de força muscular melhoram o desempenho nas AVD (Mariano et al., 2013).

Graef, Pinto, Alberton, Lima e Kruel (2010) analisaram os efeitos do treinamento resistido realizado na água na força muscular em idosas. $O$ treino consistiu em exercícios aeróbicos e exercícios de resistência muscular localizada por 50 minutos, duas vezes por semana, durante 12 semanas. O programa incluiu 4 mesociclos de 3 semanas, respectivamente, 4 séries de 15 repetições, 4 séries de 12 repetições, 5 séries de 10 repetições e 5 séries de 8 repetições de flexão horizontal do ombro em velocidade máxima, com o uso de equipamentos resistivos. Os autores encontraram melhora na força muscular mensurada através de uma repetição máxima (1RM) foi medida no exercício crucifixo na máquina. Apesar do presente estudo ter aferido a força muscular através de dinamometria, também foi observada melhora nos níveis de FMD e FME. Isso sugere que as intervenções em meio aquático podem afetar positivamente a força muscular, que está relacionada com a autonomia funcional e a qualidade de vida de idosos, sendo importante manter um estilo de vida ativo (Guedes, Hatmann, Martini, Borges, \& Bernardelli Jr, 2012).

Vale et al. (2017b) avaliaram os efeitos da hidroginástica sobre os níveis séricos de IGF-1 e AF em 28 mulheres idosas. Assim como na presente pesquisa, o programa de treinamento foi realizado com frequência de 3 vezes por semana, por um período de 12 semanas e sessões com 50 minutos de duração. Os autores encontraram aumento significativo ( $p$ $<0,05$ ) nos níveis de IGF-1 sérico após a intervenção. O IGF-1 fornece informações acerca da densidade mineral óssea (Snow et al., 2000) e exerce efeito anabólico sobre os osteoblastos, aumentando a proliferação celular e estimando a síntese da matriz mineral óssea (Olney, 2003). Dessa forma, conforme a correlação encontrada, é possível sugerir que as atividades aquáticas de baixo impacto podem promover alterações séricas, permitindo afirmar que o metabolismo ósseo é estimulado positivamente.

Dentre as limitações do presente estudo, estão a utilização de uma amostra com apenas indivíduos do sexo feminino e o pequeno tamanho amostral, o que pode limitar a validação externa dos resultados. No entanto, a presente pesquisa estabeleceu uma composição homogênea do grupo para preservar uma validade ecológica. Outra limitação foi não ter sido realizada a aferição da circunferência abdominal, uma vez que esta medida aponta um fator de risco importante para diversas doenças cardiometabólicas.

\section{Conclusões}

A correlação entre FMD e FME, entre FMD e FME e IGF1 e IGFBP3, e entre IGF1 e IGFBP3 foi positiva e significativa, mostrando que quanto maior a força muscular de preensão manual, maior a expressão do IGF1 e do IGFBP3. Assim sendo, a intervenção com exercícios resistidos aquáticos pode melhorar os níveis de força muscular de preensão manual, de IGF-1 e de IGFBP3 em idosas com excesso de peso. Portanto, sugere-se a implementação do treinamento de força em projetos sociais que envolvam exercícios físicos e recreativas em meio aquático 
para idosos, devido aos resultados positivos encontrados no presente estudo.

\section{Contribuição e implicações práticas}

A presente pesquisa apresentou a correlação entre FMD, FME, FMMII, IGF-1, IGFBP3 e AF em idosas com excesso de peso submetidas a exercícios resistidos aquáticos. Os procedimentos metodológicos adotados para análise da força muscular e AF têm uma pronunciada aplicabilidade prática em academias e centros de condicionamento e/ou reabilitação de idosos.

\section{Agradecimentos}

Os autores agradecem as participantes do Projeto Terceira Idade em Movimento e ao Laboratório de Fisiologia do Exercício, da Universidade Estácio de Sá (Cabo Frio - RJ) pela colaboração para realizar esta pesquisa.

\section{Referências}

Alexander, N. B., Ulbrich, J., Raheja, A., \& Channer, D. (1997). Rising from the floors in older adults. Journal of the American Geriatrics Society, 45(5), 564-569.

American College of Sports Medicine (ACSM), Chodzko-Zajko, W., Proctor, D. N., Singh, M. A. F., Minson, C. T., Nigg, G. R., Salem, G. J., Skinner, J. S. (2009). Exercise and physical activity for older adults. Medicine \& Science in Sports \& Exercise, 41(7), 1510-1530.

Andreotti, R. A., \& Okuma, S. S. (1999). Validação de uma bateria de testes de atividades da vida diária para idosos fisicamente independentes. Revista Paulista de Educação Física, 13(1), 46-66.

Bastos, C. L. B., Miranda, H., Vale, R. G. S., Portal, M. N., Gomes, T. M., Novaes, J. S., \& Winchester, J. B. (2013). Chronic effect of static stretching on strength performance and basal serum IGF-1 levels. Journal of Strength \& Conditioning Research, 27(9), 2465-2472.

Borde, R., Hortobágyi, T., \& Granacher. U. (2015). Dose-response relationships of resistance training in healthy old adults: A systematic review and meta-analysis. Sports Medicine, 45(12), 1693-1720.

Borg, G. A. (1982). Psychophysical bases of perceived exertion. Medicine \& Science in Sports \& Exercise, 14(5), 377-381.

Castro, J. B. P., \& Vale, R. G. S. (2017). Insulin-like growth factor I (IGF1) in older adults: a review. MOJ Gerontology \& Geriatrics, 1(6), 175-176.

Cappola, A. R., Bandeen-Roche, K., Wand, G. S., Volpato, S., \& Fried, L. P. (2001). Association of IGF-I levels with muscle strength and mobility in older women. Journal of Clinical Endocrinology \& Metabolism, 86(9), 4139-4146.

Daniel, F. N. R., Vale, R. G. S., Giani, T. S., Bacellar, S., \& Dantas, E. H. M. (2012). Functional autonomy of elderly women enrolled in a physical activity program. Acta Scientiarum. Health Sciences, 34(3), 151-156.

Dantas, E. H. M., \& Vale, R. G. S. (2004). Protocolo GDLAM de avaliação da autonomia funcional. Fitness \& Performance Journal, 3(3), 175180.

Dantas, E. H. M., Salomão, P. T., Vale, R. G. S., Achour Júnior, A., Simão, R., \& Figueiredo, N. M. A. (2008). Escala de esforço percebido na flexibilidade (PERFLEX): um instrumento adimensional para se avaliar a intensidade? Fitness \& Performance Journal, 7(5), 289-294.

Dantas, E. H. M., Figueira, H. A., Emygdio, R. F., Vale, R. G. S. (2014). Functional autonomy GDLAM protocol classification pattern in elderly women. Indian Journal of Applied Research, 4(7), 262-266.

Desrosiers, J., Bravo, G., Hébert, R., \& Dutil, E. (1995). Normative data for grip strength of elderly man and woman. American Journal of Occupational Therapy, 49, 637-644.

Eliakim, A., Oh, Y., \& Cooper, D. M. (2000). Effect of single wrist exercise on fibroblast growth factor-2, insulin-like growth factor, and growth hormone. American Journal of Physiology Regulatory, Integrative and Comparative Physiology, 279(2), R548-R553.

Fornelli, G., Isaia, G. C., \& D’Amelio, P. (2016). Ageing, muscle and bone. Journal of Gerontology and Geriatrics, 64(3), 75-80.

Friedrich, N., Haring, R., Nauck, M., Lüdemann, J., Rosskopf, D., SpilckeLiss, E., Felix, S. B., Dörr, M., Brabant, G., Völzke, H., Wallaschofski, H. (2009). Mortality and serum insulin-like growth factor (IGF)-I and IGF binding protein 3 concentrations. Journal of Clinical Endocrinology \& Metabolism, 94(5), 1732-1739.

Frontera, W., Hughes, V., Fielding, R., Fiatarone, M., Ewans, W., \& Roubenoff, R. (2000). Aging of skeletal muscle: a 12-year longitudinal study. Journal of Applied Physiology, 88(4), 1321-1326.

Frystyk, J. (2010). Exercise and the growth hormone-insulin-like growth factor axis. Medicine \& Science in Sports \& Exercise, 42(1), 58-66.

Graef, F. I., Pinto, R. S., Alberton, C. L., Lima, W. C., \& Kruel, L. F. (2010). The effects of resistance training performed in water on muscle strength in the elderly. Journal of Strength \& Conditioning Research, 24(11), 3150-3156.

Grimberg, A., \& Cohen, P. (2000). Role of insulin-like growth factors and their binding proteins in growth control and carcinogenesis. Journal of Cellular Physiology, 183(1):1-9.

Guedes, D. P., Hatmann, A. C., Martini, F. A. N., Borges, M. B., \& Bernardelli Jr, R. (2012). Quality of life and physical activity in a sample of Brazilian older adults. Journal of Aging and Health, 24(2), 212-226.

Guralnik, J. M., Simonsick, E. M., Ferrucci, L., Glynn, R. J., Berkman, L. F., Blazer, D. G., Scherr, P. A., Wallace, R. B. (1994). A short physical performance battery assessing lower extremity function: association with self-reported disability and prediction of mortality and nursing home admission. Journal of Gerontology, 49(2), 85-94.

Hale, L. A., Waters, D., \& Herbison, P. (2012). A randomized controlled trial to investigate the effects of water-based exercise to improve falls risk and physical function in older adults with lower-extremity osteoarthritis. Archives of Physical Medicine and Rehabilitation, 93(1), 27-34

Hillman, T. E., Nunes, Q. M., Hornby, S. T., Stanga, Z., Neal, K. R., Rowlands, B. J., Allison, S. P., \& Lobo, D. N. (2005). A practical posture for hand grip dynamometry in the clinical setting. Clinical Nutrition, 24(2), 224-228.

Huayllas, M. K. P., Carvalhaes-Neto, N., Ramos, L. R., Kater, C. E. (2001). Níveis séricos de hormônio de crescimento, fator de crescimento símile à insulina e sulfato de deidroepiandrosterona em idosos residentes na comunidade. Correlação com parâmetros clínicos. Arquivos Brasileiros de Endocrinologia \& Metabologia, 45(2), 157166.

Johnson, B. L., \& Nelson, J. K. (1979). Practical measurements for evaluation in Physical Education. United States of America: Burgess Publishing.

Jura, M., \& Kozak, L. P. (2016). Obesity and related consequences to ageing. Age, 38(1), 23.

Kamel, H. K. (2003). Sarcopenia and aging. Nutrition Reviews, 61(5), 157-167.

Lima, V. P., Nunes, R. A. M., Castro, J. B. P., Souza, C. C., Rodrigues, F. A. B., \& Vale, R. G. S. (2017). Variações hemodinâmicas em idosas pré e pós-exercícios em hidroginástica. Revista de Educação Física, 86(1), 18-24.

Locks, R. R., Costa, T. C., Koppe, S., Yamaguti, A. M., Garcia, M. C., \& Gomes, A. R. S. (2012). Effects of strength and flexibility training on functional performance of healthy older people. Brazilian Journal of Physical Therapy, 16(3), 184-190.

Marfell-Jones, M., Olds, T., Stewart, A., \& Carter, L. (2006). International standards for anthropometric assessment. Potchefstroom, South Africa: ISAK.

Mariano, E. R., Navarro, F., Sauaia, B. A., Oliveira Junior, M. N. S., \& Marques, R. F. (2013). Força muscular e qualidade de vida em idosas. Revista Brasileira de Geriatria e Gerontologia, 16(4), 805811. 
Mazini Filho, M. L., Venturini, G. R. O., Castro, J. B. P., Silveira, A. R., Souza, R. M., Mantovani Neto, J., \& Ferreira, M. E. C. (2018). Força e potência muscular para autonomia funcional de idosos: uma breve revisão narrativa. Revista de Educação Física, 87(3), 439-446.

Mota, J. F., Rinaldi, A. E. M., Pereira, A. F., Orsatti, F. L., \& Burini, R. C. (2011). Anthropometric indicators as risk markers for metabolic abnormalities. Ciência \& Saúde Coletiva, 16(9), 3901-3908.

Oliveira, L. S. C., Côrtes, G. G., Vale, R. G. S., \& Dantas, E. H. M. (2003). Níveis séricos de IGF-1 em gerontes. Fitness \& Performance Journal, 2(5), 289-291.

Olney, R. C. (2003). Regulation of bone mass by growth hormone. Medical and Pediatric Oncology, 41(3), 228-234.

Robertson, R. J., Goss, F. L., Rutkowski, J., Lenz, B., Dixon, C., Timmer, J., Frazee, K., Dube, J., \& Andreacci, J. (2003). Concurrent validation of the OMNI perceived exertion scale for resistance exercise. Medicine \& Science in Sports \& Exercise, 35(2), 333-341.

Rosenberg, I. H. (1997). Sarcopenia: origins and clinical relevance. Journal of Nutrition, 127(5), 990S-991S.

Sipilä, S., Multanen, J., Kallinen, M., Era, P., \& Suominen, H. (1996). Effects of strength and endurance training on isometric muscle strength and walking speed in elderly women. Acta Physiologica, 156(4), 457-464.

Snow, C. M., Rosen, C. J., \& Robinson, T. L. (2000). Serum IGF-I is higher in gymnasts than runners and predicts bone and lean mass. Medicine \& Science in Sports \& Exercise, 32(11), 1902-1907.

Steib, S., Schoene, D., \& Pfeifer, K. (2010). Dose-response relationship of resistance training in older adults: A meta-analysis. Medicine \& Science in Sports \& Exercise, 42(5), 902-914.

Vale, R. G. S., Novaes, J. S., \& Dantas, E. H. M. (2005). Effects of strength and flexibility training on autonomy of older women. Revista Brasileira de Ciência e Movimento, 13(2), 33-40.

Vale, R. G. S., Pernambuco, C. S., Novaes, J. S., \& Dantas, E. H. M. (2006) Teste de autonomia funcional: vestir e tirar uma camiseta (VTC). Revista Brasileira de Ciência e Movimento, 14(3), 71-78.

Vale, R. G. S., \& Dantas, E. H. M. (2008). Autonomia funcional do idoso. In: Dantas, E. H. M., \& Vale, R. G. S. (Orgs.). Atividade física e envelhecimento saudável. Rio de Janeiro: Shape.

Vale, R. G. S., Oliveira, R. D., Pernambuco, C. S., Meneses, Y. P. S. F., Novaes, J. S.; Andrade, A. F. D. (2009). Correlation between basal serum IGF-1 levels and functional autonomy in elderly women. Revista Internacional de Ciencias del Deporte, 5(14), 11-18.

Vale, R. G. S., \& Rodrigues, V. F. (2014). Efectos del entrenamiento de fuerza sobre los niveles de IGF-1 y autonomía funcional de adultos mayores. Revista Ciencias de la Actividad Física UCM, 15(2), 35-42.

Vale, R. G. S., Ferrão, M. L. D., Nunes, R. A. M., Silva, J. B., Nodari Júnior, R. J., \& Dantas, E. H. M. (2017a). Muscle strength, GH and IGF-1 in older women submitted to land and aquatic resistance training. Revista Brasileira de Medicina do Esporte, 23(4), 274-279.

Vale, R. G. S., Castro, J. B. P., Oliveira, R. D., Pernambuco, C. S., Oliveira, F. B., Mattos, R. S., Gama, D. R. N., Nunes, R. A. M., \& Dantas, E. H. M. (2017b). Effects of hydrogymnastics on IGF-1 and functional autonomy in elderly women. MOJ Gerontology \& Geriatrics, 1(5), 142-147.

Vale, R. G. S., Castro, J. B. P., Mattos, R. S., Rodrigues, V. F., Oliveira, F. B., Gama, D. R. N., Rosa, G., \& Nunes, R. A. M. (2018). Analysis of balance, muscle strength, functional autonomy, and quality of life in elderly women submitted to a strength and walking program. Journal of Exercise Physiology Online, 21(3), 13-24.

Wagner, P. R., Ascenço, S., \& Wibelinger, L. M. (2014). Hand grip strength in the elderly with upper limbs pain. Revista Dor, 15(3), 182-185.

World Health Organization (2000). Obesity: preventing and managing the global epidemic: report of a World Health Organization Consultation. Geneva: WHO. 